\title{
Wider Research Applications of Dynamic Consent
}

\author{
Arianna Schuler Scott ${ }^{1}$, Michael Goldsmith ${ }^{1}$, and Harriet Teare ${ }^{2}$ \\ 1 Department of Computer Science, University of Oxford, UK \\ 2 Centre for Health, Law and Emerging Technologies, Nuffield Department for \\ Population Health, University of Oxford, UK
}

\begin{abstract}
As research processes change due to technological developments in how data is collected, stored and used, so must consent methods. Dynamic consent is an online mechanism that allows research participants to revisit consent decisions they have made about how their data is used. Emerging from the context of bio-banking where research data is derived from biological samples, dynamic consent has been designed to address problems with participant engagement and oversight. Through discussion that emerged during a workshop run at the IFIP 2018 Summer School, this paper explores wider research problems could be addressed by dynamic consent. Themes of design, expectation management and trust emerged, suggesting that there are overarching research problems which could be addressed with a longer term view of how research data is used, even if that use is unknown at the point of collection. We posit that the existing model of dynamic consent offers a practical research approach outside of bio-banking.
\end{abstract}

Keywords: Consent · Engagement - Revocation - Trust · Digital rights - Research practice - Data use - Cybersecurity

\section{Introduction}

Medical research relies on human subjects for cutting edge research and worldchanging discoveries. Some studies require active human participation while others do not, specifically those using biological samples donated by patients and participants alike. Biobanks are research frameworks that store biological samples and data derived from those samples for research use.

Consent, or permission to use an individual's data is a fundamental tenet of human participatory research - identifiable personal information such as biological data must be protected and responsibility for this protection lies with both research teams and the institutions behind them. Informed consent is given by a research participant in response to information communicated about a research study's associated risk, benefits and procedure. This informed decision is made with a complete understanding of what is being agreed to - the individual must be aware of their decision to take part in the research process.

Legal provision, ethical oversight and technical controls have been used to provide this protection and as technology has developed, the way these mechanisms interact within research is changing. Dynamic consent is a real-time implementation of consent focusing on engaging participants with the research process and allowing them to revoke consent for data to be used for research purposes. 
This paper explores whether there may be wider applications of dynamic consent than the context of bio-banking. By asking those involved in research about participant consent decisions and research expectations, we provide some idea of the problems experienced by researchers around engagement and revocation. This paper considers whether dynamic consent provides a solution to such problems, grounding arguments in discussion arising from the "Exploring Attitudes to Dynamic Consent in Research" workshop delivered at the 2018 IFIP Summer School. We begin with a short review of dynamic consent and how it is situated in biomedical research before describing the workshop, discussing emergent themes and providing direction for further work.

\section{Background}

A tool that supports lawful sharing and re-use of data [2], dynamic consent promotes the active participation of biomedical research participants to create socially aware and impactful research. A "social licence" [3] for research means that it's value is reciprocal - dynamic consent puts control in the hands of participants [7], allows their input to feed back into research practice [6] and emphasises the importance of individual knowledge [12] and autonomy of choice [11]. Biobanks are research frameworks where data is derived from biological samples. They often implement broad consent, where participants agree to unspecified future use of their sample at the point of donation, biopsy or clinical test.

The argument for a broad form of consent is convincing because at the point of collection it is rarely known, either by biobank or donor, what a donated sample might be used for. This presents two problems: that consent is delegated to an oversight committee that is largely invisible outside of the biobank (biobank participants prioritise transparency and the societal interest of research over their own consent [5]), and a lack of choice or control in how data is used [10] or shared. Dynamic consent allows individuals to choose the level of consent they wish to give, it is not necessarily a direct alternative to broad forms of consent, as "broad" may be a level they feel comfortable with. The point of this technology is to focus on enabling a variety of consent options at the point at which a choice is made. Focus lies with individual participants making that decision rather then researchers making it for them.

\section{Method}

Author A.S. carried out interviews with participants involved in a study using dynamic consent in June and July 2018. The aim of these interviews was to gather feedback from researchers who had built dynamic consent into their study, and from participants who had taken part in that study. The workshop prompts below emerged from initial thematic analysis of a focus group with the research team and 20 participant interviews asking what they thought research consent should look like, and what their experience of it had been. Provisional themes of research expectations and consent decisions were put to workshop attendees to prompt discussion around how to practically engage individuals in how their personal data is used for research purposes.

Twelve people attended the IFIP workshop, "An Exploration of Attitudes to Dynamic Consent in Research" in August 2018, a group of postgraduate students from different disciplines, academics, researchers and policy-makers. After an icebreaker and introduction to dynamic consent, attendees split into mixed groups of four and were asked "Do expectations of research differ between researchers and participants?" which was designed to prompt discussion around 
expectations of data-use and any other wider research context. Given time to discuss this, each group presented their positions and a second question was put to the group, "What consent decisions surround individuals [as a participant in a research study] and why might they revoke their data?" which aimed to draw out specific examples of data that consent could be asked for and revoked, as well as the provisions made by those requesting access. After more discussion the group presented their thoughts and as conversation had given rise to further debate, the final part of this workshop consisted of open, chaired discussion.

Notes were taken by the chair, who also collected notes from the session. These notes were transcribed into comments and grouped under the prompt they had originally appeared under: "Why do expectations of research differ...?", "What consent decisions surround...?" and "General discussion". Thematic analysis [1] was used to find initial codes that emerged from the comments. These codes were used to search for themes which were then reviewed and defined. The wider narrative for this workshop is to consider to what degree individuals should be involved in research design and what that looks like in practice.

\section{Results}

This workshop was originally designed to validate a study in progress - the discussion that emerged from this workshop provided an opportunity for mutual learning amongst participants as to some difficulties in research practice. Three themes emerged from the discussion: research design, researcher and participant expectations and trust. Table 1 below shows examples of the comments made during the workshop.

Table 1. Example comments from IFIP Summer School workshop for each of the themes drawn out by thematic analysis.

\begin{tabular}{|l|l|l|}
\multicolumn{1}{|c|}{ Design } & \multicolumn{1}{c|}{ Expectations } \\
\hline - Communication is a & - Participant preferences & - Getting people into a \\
common problem. & need to have impact. & study requires trust. \\
- Platforms, or direct contact & - Does the participant expect & - There is a level of trust \\
to engage participants? & a quick fix? & as to how data is used. \\
- Can (lay)people understand & - People do not assume \\
the language used? & (research) data will be misused. & conflicts with their values. \\
\hline
\end{tabular}

\subsection{Research design}

Communication was identified as a common problem, with miscommunication caused by differing levels of participant engagement. A point raised here was whether communication informs or misinforms participants - that researchers must be representative in what they are communicating. Further to this, the importance of communication method rather than the information being communicated, emerged as a factor in choosing between using platforms and contacting participants directly. In the words of one attendee, "the latter presents more control and the former conveys more complex information". Designing or using a platform also carries recruitment considerations: marketing ("people need to know it exists") and accessibility requirements ("I'd ask whether participants need to be trained to use it?"). 
Revocation of consent to data sharing must be an option in research whether consent is broad or dynamic, even if participants do not use it. The advantage to using dynamic consent is that it provides an accessible, nuanced mechanism for withdrawal. Research design must account for the withdrawal process. Information needs to be accessible to non-experts, and although no specific requirement seemed to be identified, the point was made that there is an obligation to remind individuals of their participation. Communication of research goals in a way that both the team and participants understand in a similar way is difficult but, it was suggested, design of the research process alongside a communication strategy that accounts for accessibility, simplicity and clarity around participant involvement could begin to address this. Being explicit about immediate benefits encourages participation, and clarifying the project's relevance to the individual and society were recommendations that emerged around the difficulty of initial mobilisation of participants at the beginning of a study and "ownership creep" which emerged as situations where the individual or group ultimately responsible for a project either was or became unclear.

\subsection{Researcher and participant expectations}

There are long and short term expectations of a study at the point of engaging a participant such as the immediacy of feedback and project justification. Participant agency is important which means that the preferences they express must result in action if required (one concern raised was that this is not always the case, in the speaker's experience). One mitigation to these decisions that have no consequence could be setting expectations during the on-boarding process and allowing the participant to specify how often they would like feedback from the study. Early engagement is important as boredom was identified as a reason that participants might revoke consent.

At a fundamental level it is the violation of the individual that presents a problem. Participants' data has value but that value is largely unappreciated by the participants themselves, as are the potential harms of inappropriate data sharing or leakage ("someone's appointment being affected by their employer finding out" or "monetary gain of third parties" were two examples given). A lack of empirical evidence of these harms creates difficulty in setting precedent and managing expectations as to what responsible data practice looks like in research.

\subsection{Trust}

Recruiting participants to a study relies on trust. The quality of research may rely on the interaction between participant and researcher, so when using technological interventions, to what extent can a platform be trusted? There is a level of trust as to what happens with participants' personal data. Culture and background are important factors in recruiting research participants. Individuals may hold back if the study is supported by or supports a cause that they cannot or will not abide by. An example discussed was Jehovah's Witnesses and studies that might later include or use blood transfusions.

\section{Discussion}

Table 2 below shows comments categorised by D (research design), E (research expectations) and $\mathrm{T}$ (trust) grouped under existing principles of dynamic consent (engagement and revocation over time). 
Table 2. Comments grouped under an extended model of dynamic consent.

\begin{tabular}{ccc} 
Engagement & Revocation & Persistence \\
T1, T2, T3, T4, T5 & & \\
E1A, E1B, E1C, E1D, & T4, E3D, E7, E8, & E1C, E2A, E2B, E4B, \\
E1E, E2B, E3A, E4A, & D4, D6A, D7A, D11 & D7D, D7E, D9, D10, \\
E4B, D1, D2, D3, D5A, & D11 \\
D5B, D6A, D7B, D7C, & & \\
D7D, D8, D10, D11 & & \\
\hline
\end{tabular}

If an individual agrees to "wider use" of their data, this may mean that, as in biobanks, researchers may want to use that data for other purposes. This may mean sharing with third parties or sharing at a later time in some unspecified way. Recent legislative developments such as the European General Data Protection Regulation (GDPR) [4] and Data Protection Act (DPA) [?] in the United Kingdom have highlighted individual data rights to a public audience but do not directly apply to processing data for research purposes, especially if that data is anonymised. The conversation around data-sharing needs to begin at the point of consenting to a study which in the case of biobanks would be the point of consenting to data collection for non-specific research use. As biobanks collect more data and different types (genomic, diagnostic, clinical and lifestyle, for example), oversight will be needed as to who data is shared with and for what purposes.

Communicating risk around data-misuse mitigates threat ahead of time but increased technology use presents cybersecurity challenges in research practice while data-security concerns are a part of initial research design, there are more obvious priorities such as research goals and methods. Security concerns must be part of the initial stages of research that involves the use of human participants as part of a risk-based approach to data-protection. Knowing where vulnerabilities are likely to be allows research methods to be developed that mitigate them. One purpose for consent procedures is to limit this type of coercion [9], providing evidence of institutional compliance, or "good behaviour".

\section{Conclusion}

A significant issue in bio-banking is that data about individuals is being collected (or derived from biological samples) for unknown future uses. Not knowing what these uses might be means that researchers may not be able to anticipate the risks associated with their misuse. Dynamic consent is a mechanism that was created to address problems presented by broad forms of consent by focusing on engaging participants with the research process and allowing revocation of consent. From a workshop run at the IFIP Summer School that drew on the experiences of academics, researchers, students and policy-makers themes emerged around research practice, participant expectations, researcher expectations and trust. Many of these issues centre on a need to consider data-use over an extended period of time. For participants, what their involvement looks like and how they or society benefit. For researchers, the relevance of their work and how its impact is communicated to the wider world.

We suggest that the dynamic consent model has wider applications and should be extended to research contexts outside of bio-banking, as its fundamental principles of consent revocation and participant engagement over time 
provide a longer term view of how data is used and shared by the researchers collecting it. Dynamic consent is a tool that provides evidence of institutional data-protection and accommodates participant autonomy. Examples are needed of projects that use a dynamic form of consent in building research process and procedure that communicates with participants on their own terms, for the greater good.

\section{References}

1. Braun, V., Clarke, V.: Using thematic analysis in psychology. Qualitative Research in Psychology (3), 77-101 (2006). https://doi.org/https://doi.org/10.1191/1478088706qp063oa

2. Dixon, W., Spencer, K., Williams, H., Sanders, C., Lund, D., Whitley, E., Kaye, J.: A dynamic model of patient consent to sharing of medical record data,. BMJ: British Medical Journal (Online) p. 348 (2014)

3. Dixon-Woods, M., Ashcroft, R.: Regulation and the social licence for medical research,. Medicine, Health Care and Philosophy 11(4), 381-391 (2008)

4. European-Parliament, G.: Data Protection Regulation (GDPR), Official. Journal of the European (2016)

5. Hoeyer, K., Olofsson, B., , N. Lynöe, T.: Informed consent and biobanks: a population-based study of attitudes towards tissue donation for genetic research.,. Scandinavian Journal of Public Health 32(3), 224-229 (2004)

6. Javaid, M., Forestier-Zhang, L., Watts, L., Turner, A., Ponte, C., , J. Barrett, H.: The RUDY Study Platform-a novel approach to patient driven research in rare musculoskeletal diseases,. Orphanet journal of rare diseases 11(1), 150 (2016)

7. Kaye, J., Whitley, E., Lund, D., Morrison, M., Teare, H., Melham, K.: Dynamic consent: a patient interface for twenty-first century research networks,. European Journal of Human Genetics 23(2), 141 (2015)

8. Melham, K., Moraia, L., Mitchell, C., Morrison, M., Teare, H., Kaye, J.: The evolution of withdrawal: negotiating research relationships in biobanking,. Life sciences, society and policy 10(1), 16 (2014)

9. O'Neill, O.: Some limits of informed consent,. Journal of Medical Ethics 29(1), $4-7(2003)$

10. Simon, C., L'heureux, J., Murray, J., Winokur, P., Weiner, G., Newbury, E., , B. Zimmerman, L.: Active choice but not too active: public perspectives on biobank consent models.,. In: Genetics in Medicine, vol. 13, p. 821 (2011)

11. Teare, H., Hogg, J., Kaye, J., Luqmani, R., Rush, E., Turner, A., Watts, L., Williams, M., Javaid, M. K.: The RUDY study: using digital technologies to enable a research partnership,. European Journal of Human Genetics 25(7), 816 (2017)

12. Teare, H., Morrison, M., Whitley, E., Kaye, J.: Towards 'Engagement 2.0': Insights from a study of dynamic consent with biobank participants,. Digital Health (2015) 